\title{
Angiogenesis: A link to Thrombosis in Athero-thrombotic Disease
}

\author{
Edward M. Conway \\ Center for Transgene Technology and Gene Therapy, Flanders Interuniversity Institute for Biotechnology, \\ University of Leuven, Belgium
}

\section{Key Words}

Angiogenesis · Coagulation · Endothelium · VEGF . Atherosclerosis - Plaque - Thrombin - Tissue factor . Hemostasis · Platelets

\begin{abstract}
New insights to explain the clinical manifestations of atherosclerosis have resulted in a paradigm in which thrombosis is the major cause of acute coronary syndromes, peripheral arterio-vascular disease and stroke. Major efforts have been directed toward developing drugs to reduce thrombin and fibrin formation. Recent studies support a central role for angiogenesis of the atherosclerotic plaque in promoting atherothrombosis. The findings, in the face of efforts to use angiogenic agents to enhance vascular function, underline the challenge in treating atherothrombotic disease.
\end{abstract}

\section{Introduction}

Atherosclerosis continues to be the leading cause of morbidity and mortality in North America, Europe and Japan, accounting for the vast majority of acute coronary events, myocardial ischemia, peripheral vascular disease, and stroke. Lifetime risks of developing coronary artery disease exceed $30-40 \%$, highlighting the crucial importance of elucidating the mechanisms of onset, and developing new approaches for early detection, prevention and effective treatment.

For hundreds of years, atherosclerosis was believed to be a degenerative disease of the vessels, and a natural outcome of the aging process. This dogma was gradually challenged as the disease was further characterized. Anatomic pathologists in the 18th and 19th centuries noted early changes during disease progression in the intima, the presence of macrophages in lesions, evidence of plaque rupture, and altered morphology of those small vessels - the vasa vasorum - that feed the arterial wall. Theories abounded as to whether the plaques were actually pus-filled, whether there was inflammation of the arteries, and why and how thrombi were laid down on eroded or ruptured plaques. Key observations in the 1840 's and 1850's, as to the permeability of arterial vessels early during the disease, and the role of cholesterol in plaque formation, have clearly impacted on our understanding of the pathogenesis of the disease.

Dr. Edward M. Conway

University of Leuven, Center for Transgene Technology and Gene Therapy Gasthuisberg O\&N, 9th Floor,Herestraat 49 B3000, Leuven, Belgium

Tel: +32 16345 783; E-mail: ed.conway@med.kuleuven.ac.be
Fax +41613061234 E-Mail: karger@karger.ch www.karger.com

Accessible online at: www.krager.com/pht
(C) 2004 S. Karger AG, Basel 424-8832/04/0336-0241\$21.0/0 
Integration of these many novel findings by pioneers in the fields of thrombosis and vascular biology, has resulted more recently in rapid progress due to the establishment of closer ties between scientists in the fields of inflammation, immunology, hemostasis-thrombosis, fibrinolysis, and angiogenesis, coupled with the development of animal models of atherosclerosis and establishment of the molecular tools to delineate critical pathways.

Reflecting the complex nature of the disease, atherosclerosis has come to be recognized as a chronic inflammatory disease of large and medium sized arteries, the initiation of which is dependent on dysfunctional endothelium, and the progression of which is modulated by the interplay between oxidized lipids, activated endothelial cells, monocytes/macrophages, chemokines, platelets, coagulation factors, T-lymphocytes, extracellular matrix (ECM), proteases, protease inhibitors, and micro-vessels within the vessel wall. Clinical manifestations of atherosclerotic disease are most often triggered by arterial thrombosis of an atherosclerotic plaque, and thus the term "atherothrombosis" has been coined [1].

The overall goal of this review is to highlight the complex role that angiogenesis plays in atherothrombosis. A brief overview of the current paradigm that describes the key events that occur in the progression of the atherosclerotic lesion will be followed by a description of the importance of the atherosclerotic plaque in clinical disease, with evidence that plaque angiogenesis is correlated with thrombosis and acute coronary events. Molecular mechanisms of angiogenesis will be reviewed, with emphasis placed on insights applicable to atherothrombosis. Mechanistic links between angiogenesis and thrombosis will thus be highlighted. The dilemma facing clinicians wishing to treat patients with ischemic disease, in the face of data suggesting that angiogenic agents might actually promote thrombosis, will be considered. Potential sites of therapeutic intervention will finally be discussed.

\section{The Vascular Endothelium and Initiation of Atherosclerosis}

The vascular endothelium is not only a barrier between blood and tissue, providing the inner lining of a sophisticated tubular network to deliver oxygen and nutrients to tissues. It is also a dynamic and key factor in maintaining the vascular tree free of clot via natural anti-coagulant mechanisms, regulating contractility, and providing a source of chemokines and adhesion molecules to regulate leukocyte trafficking in health and disease. While several risk factors combine to induce endothelial cell dysfunction - an early event in atherosclerosis - epidemiologic and subsequent in vivo and in vitro analyses support the notion that chronic exposure of vascular endothelium to oxidized low density lipoproteins (oxLDL) plays a central role in disease progression (for review, see [2]).

The arterial vascular endothelium is exquisitely sensitive to chronic minimal injury induced by oxLDL, compounded by exposure to oxidative stress [3], smoking and infections, all of which leads to endothelial cell release of mediators that modulate vascular tone, enhance platelet activity and coagulation, augment platelet and leukocyte adhesion, and promote apoptosis [4]. Endothelial dysfunction induced by oxLDL diminishes the capacity of the endothelium to synthesize bioavailable endothelial nitric oxide (eNO), a crucial vasodilator and platelet inhibitor, and of prostacyclin (PGI2), and results in enhanced expression of the potent vasoconstrictor, endothelin-1 (ET-1). Endothelial dysfunction is also characterized by evidence of increased apoptosis, exhibited by diminished expression of anti-apoptotic factors (eg. Bcl2), and increases in pro-apoptotic proteins, such as Fas, Bax, and caspase-3. With minimal endothelial cell injury, pro-inflammatory intracellular signaling pathways are recruited, including activation of NFKB, which in turn leads to transcriptional upregulation of expression of cytokines (eg. IL-1 $\beta, \mathrm{TNF} \alpha$ ), adhesion molecules (eg. ICAM-1, VACM-1), monocyte chemoattractant protein (MCP-1), and C-reactive protein (CRP) [5], all of which tend to recruit leukocytes to the injury site, to localize the injury with platelet and fibrin deposition, and to promote wound healing through the enhanced expression of a variety of growth factors.

\section{Progression of Atherosclerosis and Plaque Formation}

With initiation of the atherosclerotic process by endothelial cell dysfunction, the stage is set for a cascade of events which centers around the monocyte/macrophage and leads to the development of the atherosclerotic plaque. OxLDL may directly stimulate monocytes from the circulation and bone marrow to migrate into the intima via the dysfunctional and permeable endothelial cell layer, using specific endothelial cell receptors (eg. CD18, ICAM1, P-selectin, VCAM-1), and monocyte receptors (eg. CD16, CD64) that have been upregulated by oxLDL and via NF B-transduced signaling pathways. This process is amplified, as oxLDL also induces the release of chemotactic factors (eg. MCP-1, MCP-5, MIP-1, M-CSF, GM-CSF) by endothelial cells and monocytes/macrophages which have migrated into the intima [6]. As monocyte differentiation into macrophages occurs in the vascular wall, macrophage-specific scavenger receptors are expressed which mediate uptake of oxLDL. It 
is these lipid-laden macrophages that transform into socalled foam cells, form fatty streaks, and serve to perpetuate the atherosclerotic process by secreting chemokines and mitogens that recruit more macrophages and promote smooth muscle cell migration and proliferation into the intima. These foam cells appear to be relatively resistant to apoptosis and can themselves proliferate in response to oxLDL, M-CSF, GM-CSF, these cytokines being secreted by the foam cells themselves and by infiltrating T-lymphocytes [7].

As long as the underlying atherogenic process persists, and endothelial dysfunction is maintained, the subendothelial accumulation of monocytes/macrophages and smooth muscle cells continues, and plaque growth progresses. These lesions are extremely heterogeneous in composition, comprised of extracellular lipid, foam cells, smooth muscle cells, neutrophils, T-lymphocytes, apoptotic and necrotic cells, and debris, separated from the vessel lumen by a fibrous cap comprising collagen, proteoglycans and smooth muscle cells. Vulnerability of the plaque to rupture and thrombose is enhanced by the presence of a thin fibrous cap, and a relatively high ratio of macrophages to smooth muscle cells. This has been attributed to the ability of macrophages to secrete matrix metalloproteinases (MMPs) (including for example, collagenase, gelatinase, stromelysin, matrilysin, gelatinase, metalloelastase, and metalloproteinase-13), which digest the ECM (synthesized by smooth muscle cells) particularly at the plaque "shoulders", facilitating ready plaque disruption (reviewed in [8]). Production of some of these MMPs occurs through cyclooxygenase (COX)-2-mediated pathways, explaining the current interest in COX-2 inhibitors as a therapeutic target.

\section{Beyond the Intima}

The media and the adventitial layers also are involved in plaque growth and arterial wall remodeling that occurs during atherosclerosis. MMPs may digest the internal elastic lamina (IEL), leading to either its expansion or disruption, triggering plaque rupture and atherothrombosis. With rupture of the IEL, the media expands, and this has been associated with outward (towards the adventitial layer) plaque growth, local hemorrhage into the plaque, and the development of arterial aneurysms. Factors that favour plaque disruption have recently been extended to include the presence of inflammation, fibrosis and atrophy in the media, and prominent adventitial inflammation and proliferation of the vasa vasorum [9].

While the vasa vasorum has often been overlooked as contributing to the atherosclerotic process, methods to directly visualize this vascular structure that normally pro- vides nutrients and oxygen to the adventitial wall of medium and large sized arteries, reveal that during atherosclerosis, infiltration of microvessels into the media, intima and plaques, originates predominantly from proliferating vasa vasorum, rather than from the lumen, although the latter may also occur [10]. In that sense, the vasa vasorum provides a means whereby inflammatory cells and plasma constituents may access the plaque from the "outside", further accelerating plaque growth.

\section{Plaque Thrombosis}

It has been well-established over the past 2 decades that atherosclerotic lesion disruption or erosion with subsequent thrombus formation are key events in the pathogenesis of acute coronary syndromes. What molecular mechanisms initiate and sustain the locally hypercoagulable state that leads to thrombus formation?

Several components of the plaque are thrombogenic. Indeed, fibrinogen, fibrin and fibrin degradation products are abundant in atheromatous lesions, as is thrombin, indicating that extravascular coagulation in the vessel wall is prominent (reviewed in [11], [12]). The lipid core is itself highly thrombogenic due to its high content of tissue factor (TF). Macrophage foam cells express high levels of TF, inhibition of which may reduce thrombus formation in a porcine coronary angioplasty model. T-lymphocytes, via CD40 ligand, activate the macrophages and induce secretion of TF. OxLDL induces synthesis of TF by monocytes/macrophages, smooth muscle cells, and endothelial cells, at least in part via activation of transcription factors NFkB, AP-1 and Egf-1. Patients with acute coronary syndromes (ACS) have elevated levels of circulating TF, and the source of this TF may be from smooth muscle cells, macrophages or endothelial cells. Recent evidence suggests that the major source of circulating TF is from the apoptotic death of macrophages from within lesions, leading to shedding of TF-laden particles, in concert with exposure of phosphatidylserine and apoptotic bodies, both of which are prothrombotic. The blood-borne pool of TF may be carried by neutrophils to platelets and injured endothelium, where it may contribute to thrombus propagation. TF expression does not go totally unchecked. Tissue factor pathway inhibitor (TFPI), which may regulate procoagulant activity, is locally produced in the atherosclerotic plaques, and in animal models, inhibition of the TF pathway with TFPI reduces plaque thrombogenicity.

Beyond the plaque itself, the chronic inflammatory state induces a procoagulant state. TF is secreted by circulating monocytes. There may also be imbalances in the fibrinolytic pathway, and alpha-2-antiplasmin and plasminogen acti- 
vator inhibitor (PAI)-1 are frequently elevated in patients with ACS, the latter which may be a predictor of plaque rupture. The function of natural anticoagulant/anti-inflammatory mechanisms are also jeopardized, as cytokines downregulate expression of thrombomodulin (TM) and the endothelial protein $C$ receptor (EPCR) on the surface of dysfunctional endothelial cells overlying plaques, thereby diminishing generation of activated protein C [13]. High circulating levels of C-reactive protein further activate monocytes and endothelial cells.

With plaque disruption or erosion, and exposure of the subendothelium to blood, thrombin is generated, inducing platelet adhesion via vWF-GpIb and collagen-GpIa interactions. Protease activated receptors (PARs) are upregulated by endothelial cells, platelets, and several cells within the plaque, enhancing platelet adhesion and aggregation and clot formation. Additional thrombin generation at the platelet surface induces fibrin clot formation, which ultimately may occlude the vessel, form a mural thrombus, or embolize.

Beyond the activity of thrombin in generating a fibrin clot and activating platelets, thrombin, often via PAR signaling, affects vessel tone, endothelial cell and smooth muscle cell migration and angiogenesis [14]. Thrombin has proinflammatory properties, upregulating leukocyte adhesion molecule expression and complement activation, thereby promoting additional dysfunction of the endothelium and further recruitment of monocytes to the intima [15].

\section{Angiogenesis and the Atherosclerotic Plaque}

In 1984, Barger reported that the intima and media of coronary atherosclerotic vessels was infiltrated with a tumor-like mass of microvessels, and showed that most of this neovascularization, restricted to regions of disease, originated from the adventitial vasa vasorum. These findings have since been substantiated, and support the concept that only a small minority of these new microvessels originate from the lumen. Furthermore, the extent of intimamedia neovascularization has been closely linked to the inflammatory reaction and infiltration with macrophages/foam cells within the plaque, processes that occur early. These microvessels, similar to those seen in tumor angiogenesis, are large, friable, irregular, leaky and tortuous. They are also prone to rupture, causing intraplaque hemorrhage [16]. Similar to coronary artery lesions, carotid plaque rupture, necrosis, hemorrhage, fibrous cap thinning, and accumulation of foam cells have been correlated with neurologic symptoms in patients with carotid artery atherosclerosis [17]. In those individuals with neurologic symptoms, microvessels invading the intima and media and plaque were derived from the vasa vasorum, and were larger, irregularly shaped, and leaky, more likely to bleed and to facilitate activation and infiltration of macrophages, T-lymphocytes and plasma proteins to the lesion, with promotion of an unstable plaque. In these clinical studies, the number of foam cells correlated with the number of plaque neovessels, which were observed at corners of the plaques and in the fibrous cap of symptomatic patients, supporting an important role for plaque angiogenesis in plaque thrombosis and clinical relevant disease. Indeed, in the past few years, studies have suggested that the strongest independent factor for plaque rupture is neovascularization.

\section{Vasa Vasorum and Plaque Formation}

Vasa vasorum normally run parallel to large and medium sized arteries, with secondary branches to surround the artery (reviewed in [9]). The precise mechanisms by which neovessel formation in the intima is initiated are not well understood. Numerous angiogenic factors have been found in atherosclerotic plaques, including VEGF, Plgf, bFGF, TGF-beta, MMPs, NO, PDGF, IL-8, platelet activating factor, heme oxygenase-1[18], and thrombin, yet these do not provide clues as to the stepwise progression. Similar to the artery lumenal endothelial cells, the vasa vasorum vascular endothelium is exposed to high levels of oxLDL and oxidative stress, with consequent and persistent alterations in function of the endothelium. As the thickness of the intima and media increases due to infiltration of monocytes/macrophages, lymphocytes and smooth muscle cells, the diffusion capacity of oxygen and nutrients from the lumen is exceeded. An angiogenic response by adventitial vasa vasorum would thus be stimulated by hypoxia and ischemia. The transcription factor, hypoxia-inducible factor (HIF-1) is upregulated, which in turn enhances expression of VEGF and other angiogenic factors by a variety of cells, including endothelial cells of the vasa vasorum, and monocytes/macrophages. Activated monocytes/macrophages also stimulate endothelial cell secretion of bFGF, VEGF and IL8 , all of which, and most prominently VEGF, further induce endothelial cell proliferation and permeability [19]. In vivo models also reveal that oxidative stress caused by excess production of reactive oxygen species induces expression of high levels of VEGF and MMPs by endothelial cells and smooth muscle cells in the atheroma [20]. Under the direction of yet-to-be-characterized cues, the vasa vasorum vessels migrate to regions of hypoxia/ischemia within the atherosclerotic lesion, a process facilitated by degradation of ECM by macrophage-secreted MMPs. Some of these MMPs (eg. MMP-9) further release angiogenic factors that 
are otherwise embedded in the ECM. The importance of MMPs in the angiogenic response is evident from studies in rabbits with atherosclerosis, where lesion regression accomplished by lowering dietary lipids, was accompanied by decreased MMP activity and decreased plaque neovascularization. Additional ECM glycoproteins, such as tenascin-C which has been implicated in cell proliferation, migration and apoptosis, are also secreted by macrophages, and in clinical studies [21], their levels in plaques correlate with plaque angiogenesis, intraplaque hemorrhage, macrophage infiltration and thrombus formation.

OxLDL in the subendothelium renders the endothelium of the new microvessels, dysfunctional, promoting a further inflammatory process, attracting more macrophages via the vasa vasorum. Plasma proteins and erythrocytes extravasate into the interstitium, promoting inflammation and extravascular coagulation. Some of these vessels thrombose, enhancing local ischemia, providing positive feedback for the angiogenic response, while others continue to proliferate and bleed. Plaque hemorrhage may decrease the stability of the plaque, while the additional influx, via these leaky vessels, of MMP-secreting foam cells, will further break down ECM components, thin the fibrous cap, rendering the plaque susceptible to rupture or erosion.

While it has been confirmed that neovascularization of the plaque originates predominantly from the vasa vasorum, it is also possible that endothelial progenitor cells might be mobilized from the bone marrow by VEGF, Plgf, and other angiogenic factors, to the adventitial space, providing a vasculogenic response (reviewed in [22], [23]). Evidence supports this as a possible mechanism in hypoxia-induced pulmonary artery adventitial remodeling [24].

\section{Links between Angiogenesis and Atherothrombosis}

Several molecular pathways establish links between angiogenesis, coagulation, hemostasis and thrombosis that are likely relevant in the setting of atherosclerosis and atherothrombosis. In mouse and primate models of vascular injury, PAR1 is upregulated in proliferating intima. Thrombin, via PARs, increases vascular endothelial permeability, endothelial cell expression of adhesion molecules, and release of PDGF and MCP-1, which in turn facilitate angiogenesis. Activation of PAR1 markedly augments expression of VEGF [25], the latter which is required for the angiogenic activity of PAR1. Thrombin also exhibits angiogenic activity in vitro (tube formation) and in vivo [26], and upregulates VEGF release by monocytes/macrophages, vascular smooth muscle cells and endothelial cells, as well as VEGF-R2 expression, possibly

Angiogenesis mediated in part by thrombin-induced upregulation of bFGF, TGF and PDGF release from platelets [27]. Recombinant thrombomodulin may inhibit thrombininduced VEGF production, and in a balloon injury model, inhibits neointimal hyperplasia, inflammation in the lesion, and thrombus formation [28]. Its role in plaque angiogenesis has not, however, been evaluated.

As discussed, platelet activation plays a major role in atherothrombus formation. Not only do platelets support the generation of thrombin, which in turn may promote VEGF production, but platelets also are a rich source of circulating VEGF, and thus, in conjunction with other platelet cytokines, such as PDGF, and TGF-beta, may play a direct role in plaque angiogenesis [29]. Furthermore, upon activation of platelets at sites of injury, platelet microparticles are shed, levels of which have been directly correlated with atherosclerotic disease. These microparticles are functionally best characterized for their ability to support the prothrombinase reaction, facilitating hemostasis. Interestingly, the lipid content of these microparticles themselves, appear to have angiogenic activity in vitro, in that they promote proliferation, migration and tube formation of cultured endothelial cells, by mechanisms independent of VEGF or FGF [30], and induce monocyte-endothelial cell interactions [31].

VEGF is upregulated in pathologic angiogenesis, and is elevated both in atheroma and in the plasma of patients with coronary artery disease and ACS. It is mitogenic for endothelial cells, enhances TF gene expression in endothelial cells, and mobilizes hematopoietic and endothelial cell precursors from the bone marrow [32]. The VEGF homologue, Plgf, potentiates the effects of VEGF in pathologic angiogenesis, is also elevated in atheroma, is a survival factor for endothelial cells and monocytes, and mobilizes hematopoietic stem cells from the bone marrow. In HUVECs, VEGF upregulates TF, which in turn will upregulate VEGF. In patients with peripheral arterial disease, plasma levels of VEGF and TF were significantly elevated and highly correlated with each other, suggesting a link between the thrombotic diathesis of these patients and the process of angiogenesis. Indeed, TF expression in rabbit atheroma directly correlates with sprouting of new vessels in the lesions. Furthermore, dietary lipid lowering decreased the thrombotic potential of ruptured atherosclerotic plaques through a decrease in TF, which occurred concomitantly with reduced angiogenesis in the vessel wall [33]. In other studies, lipid lowering by statins also reduces VEGF in hypercholesterolemic patients, further implying an association between coagulation and angiogenesis. This hypothesis has been further supported by several clinical studies demonstrating high levels of TF, VEGF and vWF in patients with risk factors for atherosclerosis or evidence of

Pathophysiol Haemost Thromb 2003/2004;33:241-248 
coronary artery disease, indicating the simultaneous presence of abnormal thrombogenesis, angiogenesis, and endothelial cell dysfunction [34].

Recent novel insights have revealed the mechanisms by which TF regulates angiogenesis [35], that appear independent of the role of TF-VIIa in triggering coagulation. TF-VIIa promotes angiogenesis through PAR2 signaling. The cytoplasmic domain of TF is normally unphosphorylated, and in that form, negatively regulates any TF-VIIaPAR2 signaling, thereby downregulating physiologic angiogenesis. In pathological angiogenesis, such as occurs in atherosclerosis, and in conjunction with inflammation-induced activation and upregulation of PAR2, the cytoplasmic domain of TF becomes phosphorylated, and TF-VIIa-PAR2 angiogenic signaling proceeds unchecked, an effect that is selectively synergized by PDGF-BB. Indeed, neither VEGF or bFGF have a direct effect on this system, although VEGF does increase TF expression. Thus, under inflammatory conditions and in the presence of angiogenic growth factors, PAR2 and TF are upregulated, whereupon they interact to escalate angiogenesis, and naturally, of thrombosis.

Although there is much evidence to suggest that VEGF may promote atherothrombosis, there is no doubt that VEGF has vasculoprotective properties. VEGF promotes endothelial cell survival by upregulating inhibitors of apoptosis, including survivin and $\mathrm{Bcl} 2$ [36]. In that respect, since apoptosis of endothelium is procoagulant, VEGF acts as an anti-coagulant. VEGF enhances endothelial cell expression of NO and PGI2, mediators recognized to protect the vessel wall, interfere with platelet activation, and inhibit leukocyte-endothelial interactions [37]. In fact, delivery of VEGF after balloon injury, reduces mural thrombus formation [38].

\section{Angiogenic/Anti-angiogenic Agents Effects on Plaque Angiogenesis?}

There has been a steady increase in interest to deliver angiogenic agents to patients for the relief of vascular ischemia, and indeed, several angiogenic factors are currently in preclinical trials for limb and myocardial ischemia. Yet, these patients most commonly have atherosclerosis with diffuse plaques, and there may be cause for caution in using these agents, which, at least based on our current understanding of the role of the vasa vasorum in plaque growth, might exacerbate plaque rupture, thereby promoting thrombosis with severe clinical sequelae.

What is the evidence to support or refute these concerns? Several factors must be considered: Plaques already contain high levels of VEGF, and may not respond to additional exogenously administered angiogenic agents, and thus these agents may actually be safe, and indeed, protective, by diminishing ischemia and promoting endothelial cell repair. The plaque growth/rupture response to angiogenic agents may also vary depending on the effects of each agent on the recruitment of endothelial progenitor cells, inflammatory cells, endothelial cell permeability, endothelial cell apoptosis (reviewed in [39]). Furthermore, it is likely that angiogenic agents may have distinct effects in different vascular beds, in different organs, under a variety of pathophysiologic conditions.

Several studies in hypercholesterolemic rabbits have confirmed that VEGF is vasculoprotective and thus atheroprotective, preventing intimal thickening and macrophage accumulation after balloon injury [38]. However, in other studies, administration of VEGF to atherosclerotic rabbits increased the rate of formation of diet-induced atherosclerotic plaques in the thoracic aorta [40]. And plaque size was frequently associated with increased vascularity of the lesions and infiltration with macrophages, implying an association with increased risk of rupture. In similar studies by the same group, apoE-/-:apo B100-/- mice were treated with low doses of VEGF over 3 weeks, and plaque macrophage and endothelial cell content increased significantly more than in controls. The predominant mechanism was believed to be via the effect of VEGF on mobilizing VEGFR1expressing monocytes/macrophages from the bone marrow to the lesions, although other complementary more local effects were also likely to contribute to the plaque growth $[40,41]$. No evidence of changes in plaque stability were presented, and thus the clinical significance may reasonably be questioned.

To evaluate the role of angiogenic agents in regulating plaque growth, Zhao et al [42] used a gene therapy approach to interfere with VEGFR-1 (also referred to as Flt1 in mice), the receptor for VEGF and for Plgf, overexpressing soluble Flt1 (sFlt1). In a mouse model of atherosclerosis, sFlt1 inhibited expression of monocyte chemoattractant protein-1 and TGF-beta, and attenuated early vascular inflammation and late atherosclerosis. Lutton et al [43] also evaluated the role of the Plgf and VEGF on plaque formation by treating atherogenic mice with anti-Flt-1 and anti-Flk1 (VEGFR-2) antibodies. The anti-Flt1 antibody similarly reduced atherosclerotic plaque growth, but notably, without effects on plaque neovascularization. Anti-inflammatory effects were attributable to reduced mobilization of bone-marrow derived myeloid progenitors, impaired infiltration of Flt-1 expressing leukocytes into the inflamed tissues, and defective activation of myeloid cells. Remarkably, anti-Flk1 antibodies failed to affect atherosclerotic plaque development. Neither anti-Flt1 nor anti-Flk1 antibodies suppressed angiogenesis in the adventitia or in atherosclerotic lesions, and although the reasons are unclear (Plgf and VEGF both are present in plaques), the lack of effect was hypothesized to be 
due to modulation by other angiogenic/antiangiogenic factors. These studies suggest that interfering with VEGF receptors predominantly affect macrophage infiltration into the plaque, rather than primarily angiogenesis. In contrast however, Moulton et al [44] examined the effects of angiostatin in apoE-/- mice, and concluded that this anti-angiogenic agent primarily inhibited plaque neovascularization, with secondary reductions in macrophage accumulation, plaque vulnerability and progression of advanced atherosclerosis. Their studies clearly support the hypothesis that neovascularization of the vasa vasorum acts as a conduit for transfer of inflammatory cells in and out of the plaque, and that a positive feedback loop between angiogenesis and inflammation is established early in the progression of atherosclerosis. Overall, it is likely that macrophage infiltration promotes angiogenesis, and that angiogenesis promotes macrophage infiltration.

\section{The Dilemma: Treating Patients with Atherosclerosis and Ischemic Disease}

The most common underlying disease affecting patients with coronary artery disease or limb ischemia, is atherosclerosis. Is it safe to treat these patients with pro-angiogenic agents? Will plaques grow, rupture, and thrombose? Conversely, can angiogenesis inhibitors be effectively used to promote plaque regression, without inhibiting collateral vessel formation and jeopardizing the integrity of arterial blood supply? Many angiogenic inhibitors are currently in clinical trials for cancer, macular degeneration, and arthritis, and there is not yet clear evidence of detrimental effects. Similarly, clinical trials with angiogenic agents for myocardial and limb ischemia, while not overwhelmingly successful, have not clearly caused plaque progression or atherothrombosis. Nonetheless, new approaches are urgently needed.

The challenge is to design therapies that are targeted to promote collateral vessel growth in the ischemic arteries, and to interfere with plaque angiogenesis and those factors that promote atherothrombosis. With the identification of tissue-specific and disease-specific pro/anti-angiogenic proteins, these goals will become realizable. Already, apparently plaque-specific expression of VEGF isoforms have been identified [45], and these and others might ultimately be amenable for therapeutic targeting. The utilization of novel model systems, such as the zebrafish, to facilitate rapid characterization of the specific mechanisms regulating microvessel growth and migration during health and disease, will provide insights that will lead to the development of new angiogenic molecules, and clues as to how the vasa vasorum is formed and proliferates. As the diversity of the vascular endothelium is explored, a clear understanding as to why certain vascular beds, such as the brachial artery, are resistant to atherosclerosis, will lead to new therapeutic approaches to effectively and safely prevent and treat atherothrombotic disease complicating atherosclerosis.

\section{References}

1 Corti R, Fuster V, Badimon JJ. Pathogenetic concepts of acute coronary syndromes. J Am Coll Cardiol 2003;41:7S-14S.

2 Kadar A, Glasz T. Development of atherosclerosis and plaque biology. Cardiovasc Surg 2001;9:109121.

3 Loscalzo J. Oxidant stress: a key determinant of atherothrombosis. Biochem Soc Trans 2003;31:1059-1061.

4 Durand E, Scoazec A, Lafont A, et al. In vivo induction of endothelial apoptosis leads to vessel thrombosis and endothelial denudation: a clue to the understanding of the mechanisms of thrombotic plaque erosion. Circulation 2004;109:25032506

5 Verma S, Yeh ET. C-reactive protein and atherothrombosis--beyond a biomarker: an actual partaker of lesion formation. Am J Physiol Regul Integr Comp Physiol 2003;285:R1253-6; discussion R7-8.

6 Schober A, Bernhagen J, Thiele M, et al. Stabilization of atherosclerotic plaques by blockade of macrophage migration inhibitory factor after vascular injury in apolipoprotein E-deficient mice. Circulation 2004:109:380-385.
Takahashi K, Takeya M, Sakashita N. Multifunctional roles of macrophages in the development and progression of atherosclerosis in humans and experimental animals. Med Electron Microsc 2002;35:179-203.

8 Lafont A. Basic aspects of plaque vulnerability. Heart 2003;89(10):1262-2167.

Hayden MR, Tyagi SC. Vasa vasorum in plaque angiogenesis, metabolic syndrome, type 2 diabetes mellitus, and atheroscleropathy: a malignant transformation. Cardiovasc Diabetol 2004;3(1):1.

10 Corti R, Fuster V. New understanding, diagnosis, and prognosis of atherothrombosis and the role of imaging. Am J Cardiol 2003;91(3A):17A-26A.

11 Rauch U, Osende JI, Fuster V, Badimon JJ, Fayad $\mathrm{Z}$, Chesebro JH. Thrombus formation on atherosclerotic plaques: pathogenesis and clinical consequences. Ann Intern Med 2001;134(3):224-238.

12 Viles-Gonzalez JF, Badimon JJ. Atherothrombosis: the role of tissue factor. Int J Biochem Cell Biol 2004:36:25-30.

13 Laszik ZG, Zhou XJ, Ferrell GL, Silva FG, Esmon CT. Down-regulation of endothelial expression of endothelial cell protein $\mathrm{C}$ receptor and thrombomodulin in coronary atherosclerosis. Am J Pathol 2001;159:797-802.
14 Tracy RP. Thrombin, inflammation, and cardio- vascular disease: an epidemiologic perspective. Chest 2003;124:49S-57S.
$>15$ Major CD, Santulli RJ, Derian CK, Andrade- Gordon P. Extracellular mediators in atherosclero- sis and thrombosis: lessons from thrombin recep- tor knockout mice. Arterioscler Thromb Vasc Biol 2003;23:931-939.
-16 Ross JS, Stagliano NE, Donovan MJ, Breitbart RE, Ginsburg GS. Atherosclerosis: a cancer of the blood vessels? Am J Clin Pathol 2001;116:S97- 107.
17 McCarthy MJ, Loftus IM, Thompson MM, et al. Angiogenesis and the atherosclerotic carotid plaque: an association between symptomatology and plaque morphology. J Vasc Surg 1999;30:261- 268.
18 Bussolati B, Ahmed A, Pemberton H, et al. Bifunctional role for VEGF-induced heme oxyge- nase-1 in vivo: induction of angiogenesis and inhi- bition of leukocytic infiltration. Blood 2004;103:761-766.
19 Pakala R, Watanabe T, Benedict CR. Induction of endothelial cell proliferation by angiogenic factors released by activated monocytes. Cardiovasc Radiat Med 2002;3:95-101.


20 Khatri JJ, Johnson C, Magid R, et al. Vascular oxidant stress enhances progression and angiogenesis of experimental atheroma. Circulation 2004;109:520-525.

21 Kenji K, Hironori U, Hideya Y, Michinori I, Yasuhiko H, Nobuoki K. Tenascin-C is associated with coronary plaque instability in patients with acute coronary syndromes. Circ J 2004;68:198203.

22 Iyer S, Acharya KR. Role of placenta growth factor in cardiovascular health. Trends Cardiovasc Med 2002;12:128-134.

23 Nagy JA, Dvorak AM, Dvorak HF. VEGFA(164/165) and PlGF: roles in angiogenesis and arteriogenesis. Trends Cardiovasc Med 2003;13:169-175.

24 Davie NJ, Crossno JT, Jr., Frid MG, et al. Hypoxia-induced pulmonary artery adventitial remodeling and neovascularization: contribution of progenitor cells. Am J Physiol Lung Cell Mol Physiol 2004;286:L668-678.

25 Yin YJ, Salah Z, Maoz M, et al. Oncogenic transformation induces tumor angiogenesis: a role for PAR1 activation. Faseb J 2003;17:163-174.

26 Caunt M, Huang YQ, Brooks PC, Karpatkin S. Thrombin induces neoangiogenesis in the chick chorioallantoic membrane. J Thromb Haemost 2003;1:2097-2102.

27 Bassus S, Herkert O, Kronemann N, et al. Thrombin causes vascular endothelial growth factor expression in vascular smooth muscle cells: role of reactive oxygen species. Arterioscler Thromb Vasc Biol 2001;21:1550-1555.

28 Li JM, Singh MJ, Itani M, et al. Recombinant human thrombomodulin inhibits arterial neointimal hyperplasia after balloon injury. J Vasc Surg 2004;39:1074-1083.
29 Arisato T, Hashiguchi T, Sarker KP, et al. Highly accumulated platelet vascular endothelial growth factor in coagulant thrombotic region. J Thromb Haemost 2003;1:2589-2593.

30 Kim HK, Song KS, Chung JH, Lee KR, Lee SN. Platelet microparticles induce angiogenesis in vitro. Br J Haematol 2004;124:376-384.

31 Barry OP, Pratico D, Savani RC, FitzGerald GA. Modulation of monocyte-endothelial cell interactions by platelet microparticles. J Clin Invest 1998;102:136-144.

32 Makin AJ, Chung NA, Silverman SH, Lip GY Vascular endothelial growth factor and tissue factor in patients with established peripheral artery disease: a link between angiogenesis and thrombogenesis? Clin Sci (Lond) 2003;104:397-404.

33 Jeanpierre E, Le Tourneau T, Six I, et al. Dietary lipid lowering modifies plaque phenotype in rabbit atheroma after angioplasty: a potential role of tissue factor. Circulation 2003;108:1740-1745.

34 Felmeden DC, Spencer CG, Chung NA, et al. Relation of thrombogenesis in systemic hypertension to angiogenesis and endothelial damage/dysfunction (a substudy of the Anglo-Scandinavian Cardiac Outcomes Trial [ASCOT]). Am J Cardio 2003;92:400-405.

35 Belting M, Dorrell MI, Sandgren S, et al. Regulation of angiogenesis by tissue factor cytoplasmic domain signaling. Nat Med 2004;10:502 509.

36 Zachary I. Signaling mechanisms mediating vascular protective actions of vascular endothelial growth factor. Am J Physiol Cell Physiol 2001;280:C1375-1386.

37 Dimmeler S, Haendeler J, Zeiher AM. Regulation of endothelial cell apoptosis in atherothrombosis. Curr Opin Lipidol 2002;13:531-536.
38 Khurana R, Shafi S, Martin J, Zachary I. Vascular endothelial growth factor gene transfer inhibits neointimal macrophage accumulation in hypercholesterolemic rabbits. Arterioscler Thromb Vasc Biol 2004;24:1074-1080.

39 Moulton KS. Plaque angiogenesis and atherosclerosis. Curr Atheroscler Rep 2001;3:225-233.

40 Celletti FL, Hilfiker PR, Ghafouri P, Dake MD Effect of human recombinant vascular endothelial growth factor165 on progression of atherosclerotic plaque. J Am Coll Cardiol 2001;37:2126-2130.

41 Celletti FL, Waugh JM, Amabile PG, Brendolan A, Hilfiker PR, Dake MD. Vascular endothelial growth factor enhances atherosclerotic plaque progression. Nat Med 2001;7:425-429.

42 Zhao Q, Egashira K, Inoue S, et al. Vascular endothelial growth factor is necessary in the development of arteriosclerosis by recruiting/activating monocytes in a rat model of long-term inhibition of nitric oxide synthesis. Circulation 2002;105:1110-1115.

43 Luttun A, Tjwa M, Moons L, et al. Revascularization of ischemic tissues by PlGF treatment, and inhibition of tumor angiogenesis, arthritis and atherosclerosis by anti-Flt1. Nat Med 2002;1:1.

44 Moulton KS, Vakili K, Zurakowski D, et al. Inhibition of plaque neovascularization reduces macrophage accumulation and progression of advanced atherosclerosis. Proc Natl Acad Sci U S A 2003;100:4736-4741.

45 Bhardwaj S, Roy H, Gruchala M, et al. Angiogenic responses of vascular endothelial growth factors in periadventitial tissue. Hum Gene Ther 2003;14:1451-1462. 